\title{
Detection of Rib Shadows in Digital Chest Radiographs
}

\author{
S. Sarkar ${ }^{1}$ and S. Chaudhuri ${ }^{2, \star}$ \\ ${ }^{1}$ School of Biomedical Engineering, \\ ${ }^{2}$ Department of Electrical Engineering, \\ Indian Institute of Technology, Powai, Bombay-400076, India.
}

\begin{abstract}
We propose a method for detection of rib shadows in chest radiographs that uses the knowledge of human anatomy of the thorax. Information present in the radiograph is then suitably extracted to enable the detection procedure. The method is simple but heuristic in nature and the implementation is quite fast. Details of the proposed method and the results are presented.
\end{abstract}

\section{Introduction}

There have been various efforts in [1]-[4] to detect the rib structure in the chest image so that the knowledge of the rib locations can be used for further processing. Some studies have also been reported relating to detection of interstitial infiltrates [5], [6], detection of pulmonary nodules [7], [8], and change detection in the heart and associated vasculature [9]. Most of these efforts have been to characterize a certain class of radiographic features and do not treat any disease as a whole. In [10], the author has considered the disease of pneumoconoisis but subsequently there has been no effort in quantifying a disease from analysis of radiographs.

Our primary effort has been to build a system by which the prognosis rate for patients suffering from pulmonary tuberculosis can be quantified [11]. This is done by processing a sequence of digital chest radiographs of the same patient and detecting tuberculous features in these images, and quantifying them [12]. The major problem in working with chest radiographs are the superimposed rib-shadows within the lung field. Filtering techniques cannot easily get around this problem without loss of some of the important information. The filtering technique used in [13] was initially tried to remove the rib structure but it leads to a significant loss of information. There have been efforts to solve this problem in [4] by considering small regions of interest in inter-rib spaces. This does not suffice for our problem as we are interested in quantifying the disease process in the entire lung field. Yue et al. [1] have tried a knowledge based Hough transform. Persoon et al. [2] located rib borders using template matching. DeSouza [3] located rib borders by taking vertical sections through the lung field to detect the rib edge points, followed by a curve fitting algorithm. Powell et al. [4] estimate the locations of the posterior ribs and the inter rib spaces by fitting shift

\footnotetext{
* Financial assistance from Alexander von Humboldt Foundation is gratefully acknowledged.
} 
invariant sinusoidal functions. Our effort has been to make the problem simpler by incorporating knowledge about the rib structure and position and by using some different image processing techniques which use statistics computed from the image in conjunction with the concepts in the above mentioned publications. In the next section, the processing of the the digital chest radiographs to locate the different anatomical regions of interest are discussed. Specifically, the lung fields are located in the image and also the clavicles are detected to provide a reference point for labeling the ribs. Subsequently we discuss the method used to locate the rib spaces.

\section{Pre-processing}

In a first view a radiologist locates the normal landmarks of the chest image, namely the lung fields, the heart shadow, the shadows of the ribs, the thoracic cage boundary, the mediastinum, the diaphragm, etc. This pre-scanning aids him to see if there is any gross abnormality and to locate the region of interest for examination. Subsequently the radiologist will look for finer details in the region of interest. We proceed in a similar way.

\subsection{Detection of lung field}

The lung field consists of a darker region of aerated lung tissue superposed by the brighter opacities of the rib structure. The extremities of the lung fields are marked by the thoracic cage at the periphery, the mediastinum at the center and the diaphragm at the lower ends. The shadow of the heart superposes near the lower end of the left lung.

Detection of thoracic cage : The methods used in $[1,4]$ were combined and the following algorithm was developed incorporating anatomical knowledge about the thoracic cage boundary.

Step 1 : All the gray level values in each column (vertical profile) are summed to obtain a horizontal signature. The horizontal signature should ideally have three peaks, one in the center corresponding to the mediastinum, and one on either side corresponding to the thoracic cage boundary.

Step 2 : Use a suitable zero-crossing detector to locate the three major local maxima in the horizontal signature. This is used as the global reference to detect and extend the cage along the vertical directions for each horizontal profile.

Step 3 : Starting from a horizontal profile at the middle of the image, one proceeds along both vertical directions to detect the zero crossings in an adapted small neighborhood of the reference positions. Interpolate using the local slope in case a proper match is not found in the neighborhood for a particular horizontal profile. The position of the thoracic cage boundary is then smoothened using an averaging template.

Detection of spinal column : The spinal column and the mediastinal region of the heart are fused together with respect to their radiopacity and their anatomical disposition. The method used to detect the location of the spinal column is adapted from the technique used by Yue et. al. [1] and is described as follows, Step 1 : The mid-line of the thoracic cage is calculated by averaging the thoracic cage boundary points in each horizontal profile. If pixels in the mid-line are inside 
a \pm 5 pixels neighborhood of the global peak then it is considered to be one of the pixels in the mid-line of the spinal column.

Step 2 : The knowledge of the size of the spinal column relative to the thorax size is used to determine the size by which the mid-line of the spinal column is to be dilated to obtain the whole of the spinal column. By taking measurements on several radiographs this ratio is found to be approximately $1: 10$.

Detection of diaphragm and heart border : The diaphragm is the lowermost edge of the lung field. The heart is manifested as a radiological opacity which is clearly visible in the mediastinum side of the lower lobe of the left lung. The diaphragm and the heart border is detected by the following procedure:

Step 1 : Average each vertical profile by a template of size approximately equal to the inter-rib distance. This removes the spatial variation due to the ribs and other smaller anatomical structures retaining the information about larger features such as the lung tissue and the abdominal tissue.

Step 2 : The point where there is maximum change of positive slope is detected for every profile. These points usually correspond to the diaphragm or the heart boundary.

Results of lung field detection : The combination of the proposed algorithms works for most of the images in the database. It does not work in presence of the gross abnormality (e.g. a lobar consolidation of the lower lobe of the right lung) as it could not be distinguished from the radiopacity of the region below the diaphragm. The results of lung field detection for two of the images are shown in Fig.1. All subsequent processing are restricted to the detected lung field.

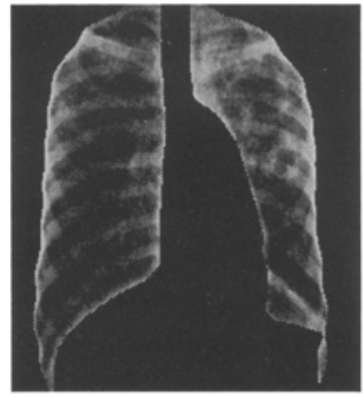

(a)

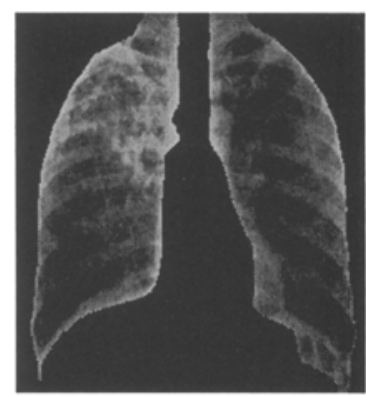

(b)

Fig. 1. Results of lung field detection for two different radiographs.

\subsection{Detection of clavicle}

The intersection of the two clavicles is used as a reference point for finding the location of different features in the image. The fourth posterior rib originates near the junction of the two clavicles. Also the orientation of the clavicle is unique from rest of the features in the respective sides of the lung. Hence we perform:

Step 1 : Each vertical profile is subjected to band pass filtering so as to remove the average variation in the gray levels due to the regions which are outside the lung field. 
Step 2 : Each filtered profile is thresholded at the $60 \%$ of the maximum value to obtain a binary image. The high states correspond to regions of high radioopacity including the clavicle.

Step 3 : The longest straight line segment having a particular orientation and lying near the top left (or right) of the binarized image is detected using the Hough transform, giving two straight line approximations of the clavicle. Fig.2 shows the detected clavicle superposed on the thresholded image and on the original image.

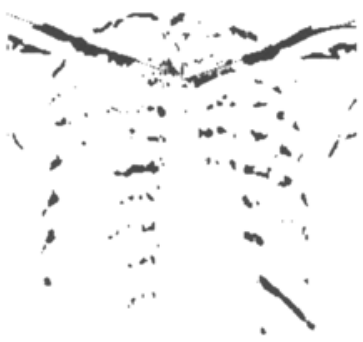

(a)

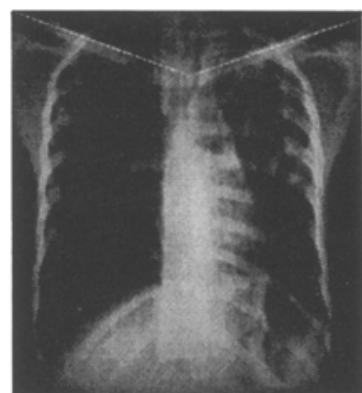

(b)

Fig. 2. Detected clavicle superposed on (a) thresholded and (b) original images.

Step 4: The location of the clavicle junction is estimated as the average of the positions where the two lines detected above intersect the mid-line of the spinal column. The location of the clavicle junction is later used as a reference point to compute the rib positions.

\section{Detection of rib spaces and inter-rib spaces}

Anatomical regions of the lung field are superposed by rib shadows causing a major impediment to using different processing techniques for detection of features. The following method classifies the lung field into the rib spaces and the inter-rib spaces incorporating anatomical information available about them. Step 1 : Each vertical profile is subjected to band pass filtering so as to remove the average variation in the gray levels due to the regions which are outside the lung field.

Step 2 : The filtered profile obtained above is then thresholded with the threshold being at zero to obtain a binary image. The high states in this thresholded profile corresponds to rib spaces and/or radiologically opaque anatomical structures. The low states in the binary image correspond to the inter-rib spaces. Fig. $3(\mathrm{a})$ illustrates the result of step 2 .

Step 3 : The binary image obtained is passed through a successive morphological closing-opening filter with a small circular element to get rid of the unwanted, isolated radiological features. Fig. 3(b) illustrates the result of step 3 .

Step 4 : Each vertical profile in the binary image is labeled such that a connected string of pixels having the high states in the binary image is given the same label. 


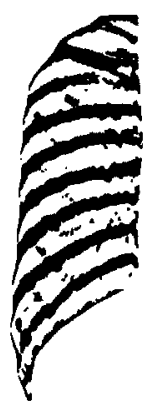

(a)
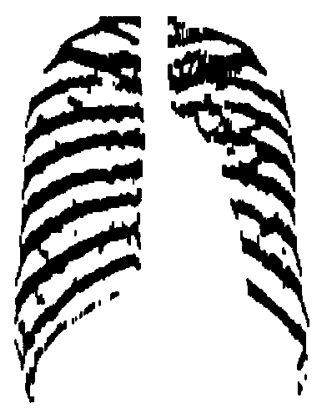

(b)

Fig. 3. (a) Binary image after thresholding operation in step 2, (b) binary image after morphological filtering.

Each rib space thereby gets a different label and this labeling information is used to calculate the statistics of the rib spaces and the inter-rib spaces.

Step 5 : The length (number of pixels) of each labeled rib space in all the vertical profiles are calculated and the histogram of the rib-space size is obtained. The mode of this distribution corresponds to the most probable rib-size.

Step 6 : Retain only those ribs (in step 3 ) whose sizes are quite close the computed value of the mode. This step in effect gets rid of the pathological features in the binary image. The resulting rib structure is shown in Fig. 4(a).

Step 7 : The mean position of the retained rib-spaces in every vertical profile is found. This gives the approximate location of the mid-line of the rib-spaces in the binary image obtained in Step 6. The mid-lines of rib-spaces is illustrated Fig. 4(b). The vertical distance between two consecutive mid-lines of ribs is called the inter-rib distance. The mean inter-rib distance is computed from the entire image.

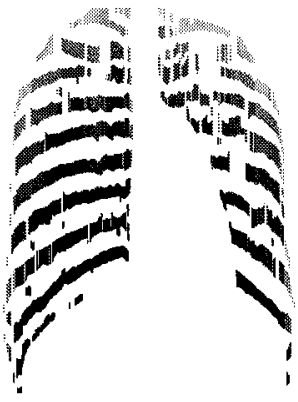

(a)

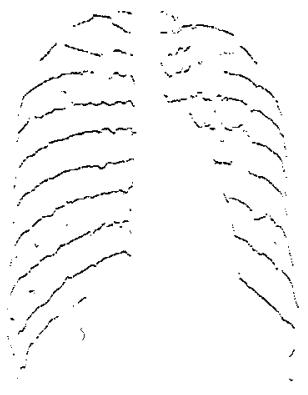

(b)

Fig. 4. (a) Image resulting from removal of segments having less probable rib space size in step 6, (b) image showing the mid-lines of rib spaces.

Step 8 : The global position of the ribs along the spinal column is located by 
projecting the mid-lines in the central part of the lung field in the horizontal direction. The detected peaks in this projection approximately correspond to the positions of the origin of the ribs from the spinal column (see Fig. 5(a)).

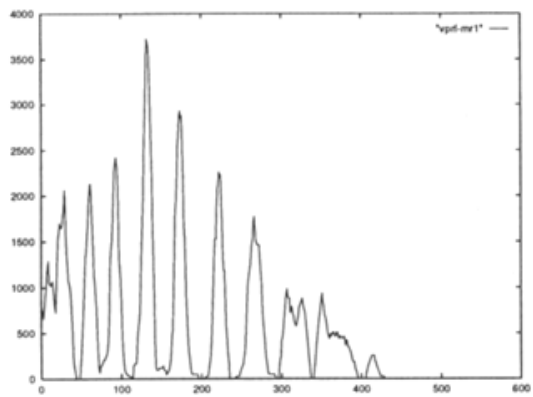

(a)

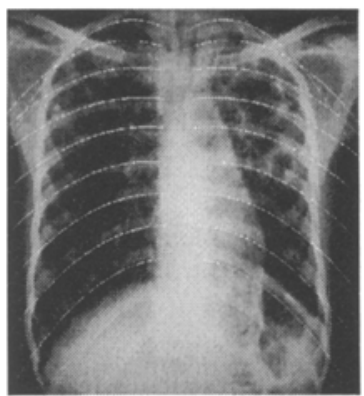

(b)

Fig. 5. (a) Horizontal projection of mid-lines for detection of rib location on the spinal column (in pixels). (b) Image showing modeled rib trajectories.

Step $9:$ The detected peaks below the clavicle junction are considered. Not all the global rib positions can be detected by the above method. The rest of the rib positions are estimated using the mean inter-rib distances and relative distance between the ribs in the spinal column which has been estimated from a study of a large number of radiographs present in our database. The relative distances are tabulated in Table 1.

\begin{tabular}{|l|c|c|c|c|c|c|c|c|c|c|}
\hline rib nos. & 1,2 & 2,3 & 3,4 & 4,5 & 5,6 & 6,7 & 7,8 & 8,9 & 9,10 & 10,11 \\
\hline rel. dist. & 0.55 & 0.675 & 0.775 & 0.85 & 0.925 & 1.00 & 1.00 & 1.05 & 1.10 & 1.20 \\
\hline
\end{tabular}

Table 1. Computed relative distances between two consecutive ribs.

Step 10 : Mid-lines of ribs are modeled individually as second order polynomials of the form: $y=a_{2} x^{2}+a_{1} x+a_{0}$. From trials on all the images the coefficients $a_{1}$ and $a_{2}$ are estimated for all possible rib positions and are given in Table 2. The coefficient $a_{0}$ will depend on the position of origin of the rib on the spinal column. The modeled mid-line gives the trajectory for a robust small neighborhood search for mid-line points of a particular rib. The computed model of the rib shadow structure is shown in Fig 5(b).

Step 11 : Following a trajectory and considering a small neighborhood of \pm 10 pixels in the vertical direction for search, mid-line points truely belonging to a particular rib is detected from the image obtained in step 7 (Fig. 4(b)).

Step 12 : Now, a least squares, second order polynomial fit is performed for the set of true points for each rib. The result of the curve fit is shown in Fig. 6(a). 


\begin{tabular}{|c|c|c|c|c|c|c|c|c|c|c|}
\hline Coeff. & rib1 & rib2 & rib3 & rib4 & rib5 & rib6 & rib7 & rib8 & rib9 & rib10 \\
\hline$a_{1}$ & -0.7 & -0.5 & -0.3 & -0.2 & -0.2 & -0.2 & -0.15 & -0.1 & -0.075 & 0.05 \\
\hline$a_{2}$ & 0.0055 & 0.0035 & 0.0025 & 0.002 & 0.00225 & 0.0025 & 0.00275 & 0.003 & 0.0035 & 0.004 \\
\hline
\end{tabular}

Table 2. The coefficients used for rib trajectory initialization.

Note the improvement obtained by comparing it with Fig. $5(b)$.

Step 13 : Dilate every curve symmetrically along vertical directions by the estimated rib size (see step 5) to obtain a particular rib shadow. Results of detection of rib shadows are given in Fig. 6(b).

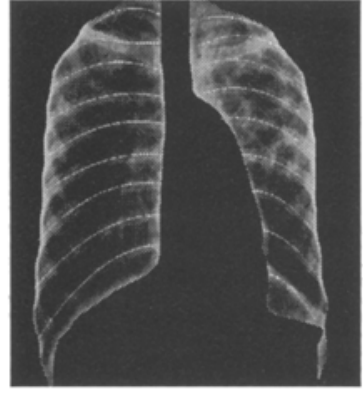

(a)
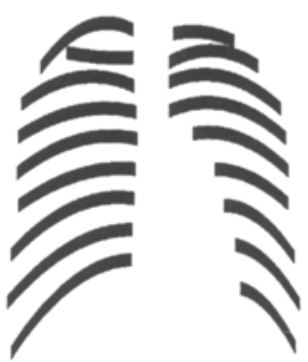

(b)

Fig. 6. (a) Image showing robust quadratic fit to detected mid-lines, and (b) the finally detected rib spaces.

\section{Results and conclusions}

The above method is applied to all the images in the database. The images that we have in our database are mainly digitized chest radiographs having features of pulmonary tuberculosis [12]. The digitization is done using an SGI workstation interfaced to a video camera. The captured image size is $500 \times 550$ pixels, maintaining the same resolution in all images. The results are reasonably accurate. The method is successful in extracting the shadows of the posterior rib structure even from the images having pathological features which are characteristics of tuberculosis. Some of the results obtained on applying this method on pathological cases are shown in Fig 7.

Some of the drawbacks inherent in this method may include improper detection of ribs supra clavicle in the image space. Also, the thickness of the rib shadows above the clavicle are significantly smaller than those below the clavicle. The relation between the sizes of the different ribs has not been incorporated in this work but can be incorporated to produce better results. 

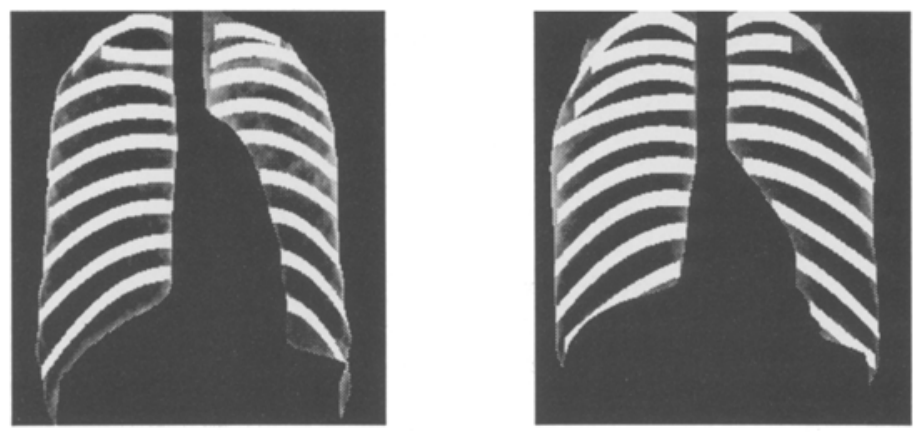

Fig. 7. Results of rib space detection.

\section{References}

1. Z. Yue, A. Goshtasby, and L. V. Ackerman, "Automatic detection of rib borders in chest radiographs", IEEE-TMI, vol 14, Sept. 1995, pp 525-536.

2. E. Persoon, "A new edge detection algorithm and its application in picture processing", Computer Graphics and Image Processing, vol 5, 1976, pp 425-446.

3. P. DeSouza, "Automated detection of ribs in chest radiographs", Computer Graphics and Image Processing, vol. 23, 1983, pp 129-161.

4. G. F. Powell, K. Doi, and S. Kasturagawa, "Localization of inter-rib spaces for lung texture analysis and computer aided diagnosis in digital chest images", Medical Physics, vol. 15(4), Jul/Aug 1988, pp. 581-587.

5. S. Kasturgawa, K. Doi, N. Nakamori, H. MacMohan, Y. Sasaki, and J. J. Fennessy, "Quantitative computer-aided analysis of lung texture in chest radiographs", Radiographics, vol. 10, 1990, pp. 257-269.

6. S. Kasturgawa, K. Doi, and H. MacMohan, "Image feature analysis and computer aided analysis in digital radiography: Detection and characterization of interstitial lung disease", Medical Physics, vol. 15(3), 1988, pp. 311-319.

7. M.Giger, K.Doi, H.MacMohan, C.Metz, and F.Yin, "Pulmonary Nodules: Computer-aided detection in digital chest images", Radiographics, vol. 10, 1990, pp.41-51.

8. M. L. Giger, N. Ahn, K. Doi, H. MacMohan, and C. E. Metz, "Computerised detection of Pulmonary Nodules in chest images: Use of morphological filters in reducing false positive detections", Medical Physics, vol. 17(5), 1990, pp. 861-865.

9. N. Nakamori, K. Doi, V. Sabeti, and H. MacMohan, "Image feature analysis and computer aided diagnosis in digital radiography: Automated analysis of sizes of heart and lung in chest images", Medical Physics, vol. 17(3), 1990, pp. 342-350.

10. R. P. Krueger, W. B. Thompson, and A. F. Turner, "Computer Diagnosis of Pneumoconiosis", IEEE Trans. on Systems, Man, and Cybernetics, vol. 10, pp. 257-269.

11. S. Sarkar and S. Chaudhuri, "Automated detection of infiltration and cavitation in digital chest radiographs of pulmonary tuberculosis", 18th Annual International Conference of IEEE-EMBS, Amsterdam, Nov. 1996.

12. S. Sarkar and S. Chaudhuri, "Evaluation and progression analysis of pulmonary tuberculosis from digital chest radiographs", Computerized Medical Imaging bo Graphics (to appear).

13. S. Chaudhuri, H. Nguyen, R.M. Rangayyan, S. Walsh, and C. B. Frank, "A Fourier domain directional filtering method for analysis of collagen alignment in ligaments", IEEE Trans. in Biomedical Engineering, July 1987, pp. 509-518. 